\title{
System View Simulation of Low-pass Signal Sampling Theorem
}

\author{
Xu Liu
}

Chongqing College of Electronic Engineering, Chongqing, 401331, China

Keywords: Sampling theorem, System View, Simulation.

\begin{abstract}
Digital transmission of analogue signal has many advantages and now has been widely applied in various fields of modern communications. Sampling theorem is the theoretical basis of digital transmission of analogue signal, and the sampling and recovery of signal play a particularly important role. This paper constructs the simulation model of the low-pass signal sampling theorem based on the software environment of System View, and conducts the simulation analysis on it to verify the sampling theorem.
\end{abstract}

\section{Introduction}

\section{System View software}

System View software is a development tool software developed by the American company, ELANIX, which is used for the design, simulation, analysis and evaluation of view system model. It adopts the graphical programming mode under the Windows environment, has a friendly and powerful debugging context, and is a power tool for the real signal-level system design and simulation [1].

The operation under the System View environment is relatively simple and clear. The simulation model can be constructed according to the system design requirements by using the various function icons offered by System View itself, and the simulation analysis can be conducted after the setting of its parameters including the time of system operation.

\section{Features of System View software}

System View is mainly used for the design and simulation of circuit and communication system, and can satisfy different levels of design from digital signal processing and filter design to complex communication system. System View in a modular and interactive interface provides users with embedded analysis engine under the Windows environment that everyone is familiar with [1-2]. When using System View, you just need to take care of the design idea and process of the project, without having to spend much of your time in programming and establishing the system simulation model. The user can complete the modeling, design and test of a complex system by just using the mouse to click on the icons without having to learn the complex computer programming or worry about whether there is a programming error in the program. System View provides a visualized and dynamic system model [1]. It uses the Token in the functional component library to represent a treating process and complete the design of the system or sub-system in the window of System View. The design process is to select Token in different component libraries from the system window and create connection in the design area, and at the same time to set the parameter of each Token to control the starting time, breaking time, and sampling frequency of the control system, and at last to analyse the results from the analysis window, thus achieving the goal of a design system and an analysis system.

\section{Digital transmission of analogue signals}

Analogue signals not only can be transmitted after carrier modulation but also can be transmitted in the way of digital communication after digitizing. The benefit provided by the digital transmission of analogue signals is that the signal-noise ratio will not become deteriorated after digital signals have gone through multiple transformations, relays, and long distance transmissions; however, analog signals will generate extra deterioration of signal-noise ratio after going through multiple relays, which reduces the quality of the transmission signals. Besides, after the analogue signals have been digitized, they can realize the time division multiplex transmission or code division 
multiple transmission, thus, can effectively improve the channel utilization. Therefore, the digital transmission technology of analogue signals has been widely applied in various fields of modern communications, and digitized analogue signals exist everywhere, from wired SPC exchange to wireless GSM mobile phone and from satellite digital TV broadcasting to long-distance optical fiber communication.

Sampling theorem is the theoretical basis of digital transmission of analogue signal. It points out that, If sampling is made over the finite-time continuous signals (analogue signals) of a broadband and the sampling rate reaches a certain value, the original signals can be recovered accurately at the receiving terminal according to those sampling values[3-4]. That is to say, transmission of analogue signals is not necessarily the transmission of transmission of analogue signals; instead, it can be achieved only by the transmission of the sampling values which have been obtained according to the sampling theorem.

\section{Establishment of the System View simulation model of low-pass sampling signals}

\section{Time domain sampling theorem}

The signals $f_{s}(t)$ obtained through the discrete sampling on continuous signals $f(t)$ have values only in some discrete moments. But when certain conditions are met, the sampling signals $f_{s}(t)$ can completely represent the original signals $f(t)$, i.e., $f_{s}(t)$ includes all information of $f(t)$. Thus, $f_{s}(t)$ transmission can be achieved without $f(t)$ direct transmission. At the system terminal (such as the receiving terminal of communicator), recovery of original signal $f(t)$ is still possible from $f_{s}(t)$ through some sort of technology.

The sampling theorem points out that, for the continuous-time signal $f(t)$ with the frequency band limited within the range of $\left(0, f_{m}\right)$, if an equal interval sampling is made on it in $T_{s} \leq \frac{1}{2 f_{m}}$

Then the signal $f(t)$ will be completely identified by the obtained sampling values $f_{s}(t)$. That means that in the case of sampling rate no less than two times of the signal broadband, signal aliasing surely will not happen. $\frac{1}{2 f_{m}}$ is the maximum sampling interval, also called Nyquist interval[3].

\section{Re-construction (recovery) of signals}

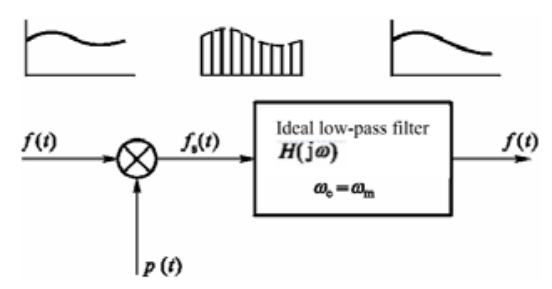

Fig. $1 f(t)$ Recovery Functional Block Diagram by Ideal Low-Pass Wave Filter

The re-construction of signals refers to the process of the original signals $f(t)$ recovered from the sampling signal $f_{s}(t)$. Since the majority of the sampled original signals have been lost and the sampling signals $f_{s}(t)$ are only a small part of $f(t)$, for the recovery of the original signals $f(t)$ from the sampling signals $f_{s}(t)$, the above mentioned time domain sampling theorem must be met. Original signals can be recovered immediately by using the ideal low-pass wave filter (Its amplitude response is a constant $\mathrm{T}$ in the pass-band and is 0 in the stop band). The cut-off angular frequency of the ideal low-pass wave filters is $\omega_{c}\left(\omega_{m}<\omega_{c} \leq \frac{\omega_{s}}{2}\right)$. Its functional block diagram is as shown in Figure 1.

It is impossible in actual projects to recover the original signals $f(t)$ without any distortion. First, since the spectrum of the real signal existing in a limited time is infinitely wide, which makes it impossible to determine the maximum frequency $f_{m}$, so it only approximately satisfies the first 
condition of the sampling theorem; secondly, the complete recovery of $f_{s}(t)$ must be subjected to the application of $f_{s}(t)$, the ideal low-pass wave filter which is however unavailable. The actual low-pass filter in the projects can only roughly meet the ideal low-pass feature. Therefore, even $F_{s}(\omega)$ has no spectral aliasing, actual filters will be able to get all the information, including $F(\omega)$. And so some distortion is inevitable in the recovery. If the maximum frequency $\omega_{m}$ is given, we normally take $\omega_{s} \geq(5 \sim 10) \omega_{m}$ in the projects; and if the signal broadband $\omega_{B}$ is given, it normally takes $\omega_{s} \geq(5 \sim 10) \omega_{B}$, and when the maximum frequency of the interfering signal is $\omega_{f}$, then $\omega_{s} \geq 2 \omega_{f}$ should be met. Figure 2 shows the schematic diagram of low-pass signal sampling and recovery.

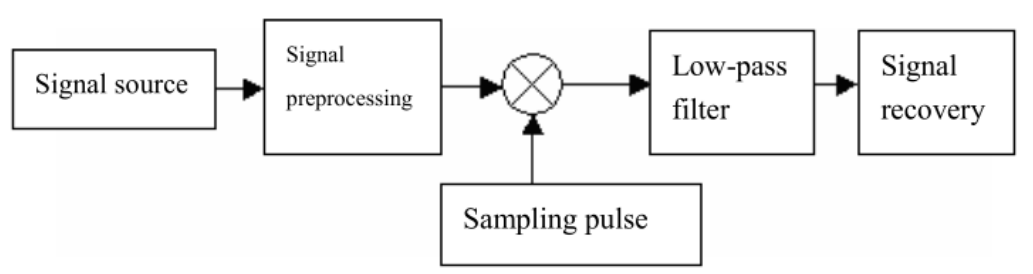

Fig. 2: Schematic Diagrams of Signal Sampling and Recovery

\section{Simulation of low-pass signal sampling}

Establishment of a simulation figure of System View. (1) Enter into System View and set the running time of the system by clicking $1-1$ on the "System Time" button at the toolbar; then set "Sample Rate" and "No. of Samples".

(2) Drag out a source icon $\boxminus$ "Source" from the symbol library to put it in the design window and double click it; in the pop-up source window, choose the sinusoidal signal "Sinusoid" in the "Periodic". Then press "Parameter" button to set "Frequency" at 100 in the parameter settings window. Thus the symbol becomes (this signal is the sampled simulation signal with an amplitude of $1 \mathrm{~V}$, frequency of $100 \mathrm{~Hz}$ sine wave); with the same procedure define a sampling pulse, making the pulse width at 1us, i.e. $\square$ (this is a narrow-width rectangular pulse with a width of 1 micron); drag out two gains and two filters from the operators library in icons library, and set parameters respectively, i.e. $[>,[>, \square, \square$ Use the third-order Butterworth low-pass filter for restoring signals; then drag out the multiplier ${ }^{2} \mathrm{X}$ (replace the sampler); finally define three reception icons. Drag three receiver icons $\square$ to the design window and double click them, defining them as "Analysis" signal receiving type 国. That is 国, 四, 四.

(3) Link the icons; press the note-creating button E to add the Note Pad as that shown in Figure 3. Character colors and fonts can be defined by right-clicks in the text box.

The newly-built System View schematic diagram of simulation system is as shown in Figure 3

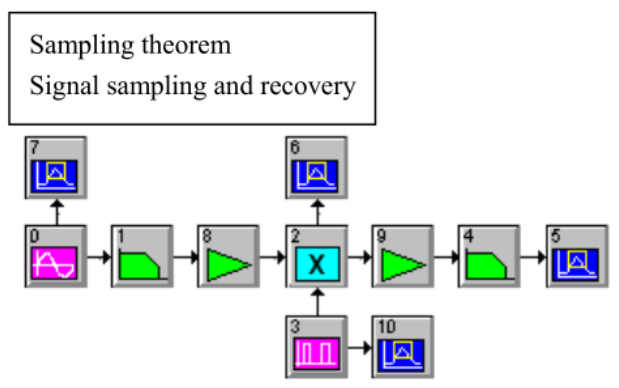

Fig. 3: Schematic Diagram of Simulation System for Signal Sampling and Recovery 
Simulation. Run the system: click the start button on the toolbar to run the system; click 鰨 "Analysis" to enter the analysis window. Taking the toolbars of the analysis window as reference, reset window arrangement according to personal preference, and modifies the sampling frequency to start the simulation analysis again. Stop simulating and save the user system. Choose "Save" in the "File" menu to save the newly made content.

In order to watch conditions for free-distortion signal sampling and recovery as well as the cause for distortion, we chose different sampling frequencies, such as $100 \mathrm{~Hz}, 200 \mathrm{~Hz}, 500 \mathrm{~Hz}$. Figure 4 shows the waveform of the original signals; Figure 5 shows the sampling pulse waveform; Figure 6 shows the waveform of $500 \mathrm{~Hz}$ after sampling; Figure 7 shows the restored waveform after passing the low-pass filter; Figure 8 and Figure 9 shows respectively restored waveforms of $100 \mathrm{~Hz}$ and 200 $\mathrm{Hz}$. Obviously, the restored waveform has greater distortion when sampling frequency is at $100 \mathrm{~Hz}$.

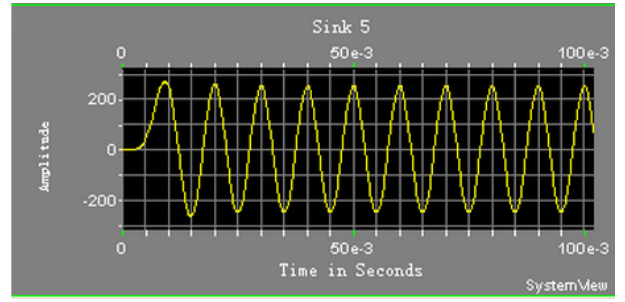

Fig. 4: The Original Waveform of Input Signals

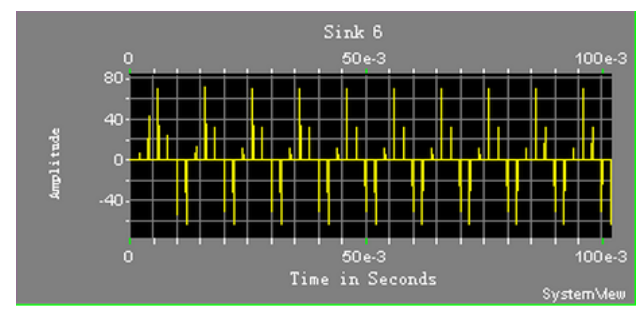

Fig. 5: Sampling Pulse Waveform

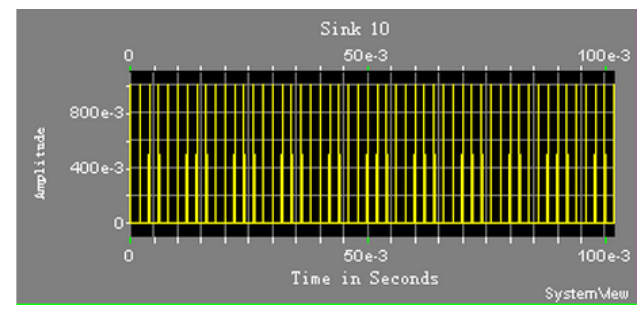

Fig. 6: $500 \mathrm{~Hz}$ After-Sampling Signal Waveform

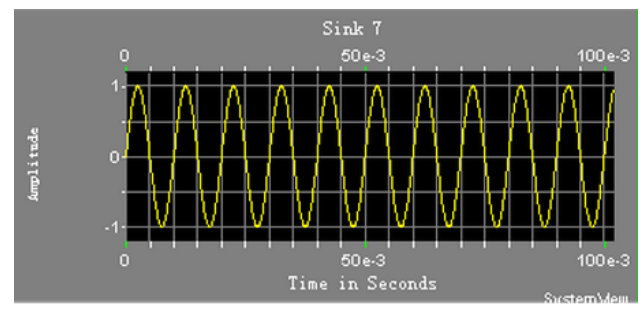

Fig. 7: 500 Hz Signal Waveform after Sampling Recovery 

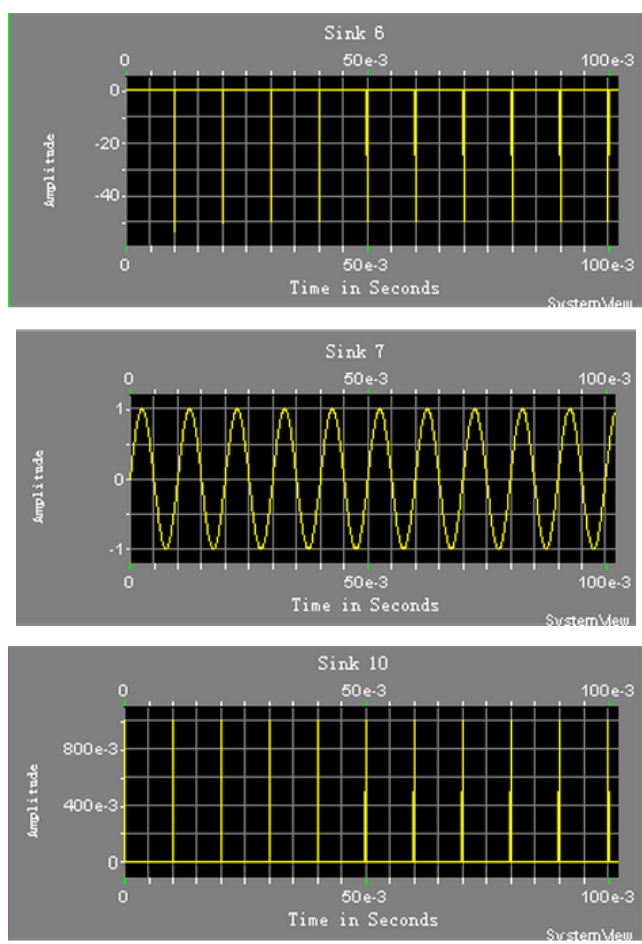

Fig.8: 100 Hz Waveform after Sampling Recovery
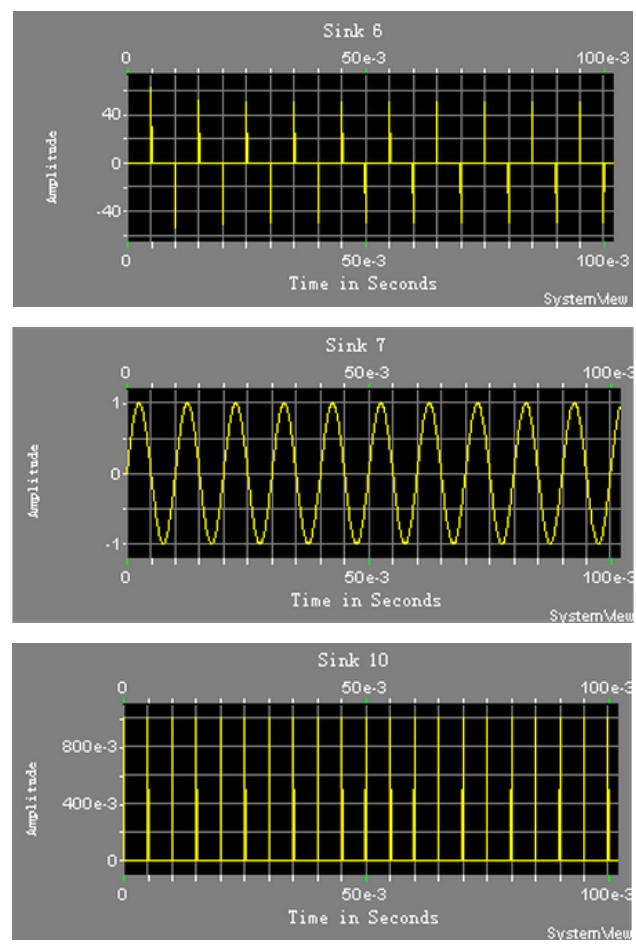

Fig. 9: $200 \mathrm{~Hz}$ Waveform after Recovery

It can be seen from the above simulation result that when the frequency is $100 \mathrm{~Hz}$, the frequency is the same as that of the original input signal, and the sampling interval is too great, which leads to loss of some elements in the original signal, thus the restored waveform (as in Figure 8) is most distorted as that of the original input signal. When the frequency is $200 \mathrm{~Hz}$, the sampling interval is the Nyquist interval, and thus the restored waveform (as in Figure 9) is much better than that in Figure 8, though still with some distortion as that of the original input signal. When the frequency is $500 \mathrm{~Hz}$, the restored signal is most accurate, with approximately the same waveform as that of the original signal. 
When the sampling frequency is below the Nyquist rate, the restored signal at the receiving end is distorted a lot due to signal aliasing; when the sampling frequency is no less than the Nyquist rate, the restored signal is roughly the same as the original signal. In theory, the ideal sampling frequency is two times the Nyquist one; but in actual projects, band-limited signals are not strictly bandlimited, and the features of actual filters are not so ideal, so the sampling frequency is usually set at 2.5-5 times $\mathrm{f}_{\mathrm{s}}$ to avoid distortion. For example, common voice signals are with a band of $3300 \mathrm{~Hz}$ or so, while sampling frequency is usually at $8 \mathrm{kHz}$.

Thus the system simulation vividly verifies the sampling theorem: sampling signals can restore the original signals under certain conditions of $f_{s} \geq 2 f_{m}$, and the higher the sampling frequency, the better the restored signal; because the spectrum of the sampling signal is a cyclical expansion of the original, the latter can be restored so long as the former passes a low pass filter with a cut-off frequency of $f_{c}\left(f_{m} \leq f_{c} \leq f_{s}-f_{m}\right)$.

\section{Conclusion}

This paper elaborates on the sampling theorem, and acquires the conditions for sampling of lowpass signals and their recovery. Theoretically, so long as the sampling theorem is followed, original signals can be restored from sampling signals. Set up a dynamic simulation model for signal sampling and recovery through the System View software, choosing different sampling frequencies, and you can get a series of simulation results which vividly verify the sampling theorem of low-pass signals.

\section{References}

[1] SunYi:System View simulation of communication development manual (Beijing. National Defence Industry Press, 2004) P.1-362

[2] Yan Qingming. Signals and System (Beijing. Higher Education Press, July, 2004) P, 94-98

[3] QingSong Cheng Daisong .Simulation and analysis of System View digital communication system (Beijing. Publishing House of Electronics Industry, 2001) P.127-136

[4] Bernard Sklar. Digital communications: Fundamentals and Applications, Xu Pingping et al. (Beijing. Publishing House of Electronics Industry, 2002) P.156-167 